\title{
ANALYSIS OF EFFICIENCY OF VACCINES AGAINST BRUCELLOSIS IN CATTLE IN THE REPUBLIC OF KAZAKHSTAN
}

\author{
ASPEN ABUTALIP ${ }^{1 *}$, NURALI MATIKHAN ${ }^{2}$, SERIK KANATBAYEV ${ }^{1}$, MARAT BAZARBAYEV ${ }^{1}$, VIKTOR VOROBYOV \\ ${ }^{1}$ Department of , Kazakh Scientific-Research Veterinary Institute, Almaty, Kazakhstan. ${ }^{2}$ Department of , Kazakh National Agrarian \\ University, Almaty, Kazakhstan. ${ }^{3}$ Department of , Veterinarian of “Vostok-Moloko" Corporation of Eastern Kazakhstan, Almaty, \\ Kazakhstan. Email: nurik200886@mail.ru
}

Received: 14 December 2016, Revised and Accepted: 01 April 2017

ABSTRACT

Objective: This article analyzes the efficiency of live vaccines obtained from the strains of Brucella abortus 82 and $75 / 79-A V$ and RB-51 in some regions of Kazakhstan in 2012-2014 and recommends optimal scheme of their use.

Methods: There were analyzed the effectiveness of the use of live vaccines (manufactured in Russia) obtained from strains of $B$. abortus 19 , 82 and 75/79-AV in combination with post-vaccination diagnostics of brucellosis in cattle in the farmsteads owned by Vostok-moloko Corporation in Eastern Kazakhstan in the period of 2012-2015.

Results: The results of these tests prove that studies of animals 1 month after the vaccination have helped to further identify the animals with hidden form of brucellosis provoked by R-vaccine, which explains the favorable outcome of the research.

Conclusion: The above-described scheme of anti-brucellosis activities ensures effective control of the epizootic process of brucellosis by establishing "permanent" (continuous) immunity and permanent control of epizooty in brucellosis threatened/unsafe herds.

Keywords: Brucellosis vaccine, Immunization, Efficiency, Anti-brucellosis measures.

(C) 2017 The Authors. Published by Innovare Academic Sciences Pvt Ltd. This is an open access article under the CC BY license (http://creativecommons. org/licenses/by/4. 0/) DOI: http://dx.doi.org/10.22159/ajpcr.2017.v10i6.16594

\section{INTRODUCTION}

Despite all the veterinary efforts on elimination of brucellosis infection in Kazakhstan, the epizootic situation with brucellosis remains disturbing. It was identified that brucellosis in cattle and small ruminants has a significant role among infectious pathology diseases in the Republic of Kazakhstan, which significantly reduces the population of livestock and negatively affects the national economy [1,2]. Scientific and practical experience shows that in the present conditions any antibrucellosis efforts for the cattle in unsafe and infection threatened areas cannot be effective enough without the use of vaccines $[3,4]$. Vaccination is aimed at the formation of immunity in animals and reduction of the risk of spread of the disease. The strategy of mass vaccination was common for Kazakhstan until 2006. The cattle was vaccinated against brucellosis with the vaccines prepared from a strain of Brucella abortus -19 and 82, while the vaccines for the small ruminants were prepared from B. melitensis strain Rev-1. From 2006 to 2012, the anti-brucellosis measures in the country were carried out without vaccination, which caused a complicated epizootic situation with brucellosis in animals. With all this in mind, it becomes relevant to study and review anti-brucellosis activities carried out in the Republic of Kazakhstan with the use of vaccination. The resulting data will help to make a justified decision about the optimal scheme of using the antibrucellosis vaccines.

\section{METHODS}

Analysis of anti-epizootic efforts for the cases of brucellosis in livestock with use of different vaccines was performed through the collection and study of data from veterinary reports of the Committee for veterinary control and surveillance under RSE "Republican Veterinary Laboratory," the KZ Ministry of Agriculture and by conducting our own epizootic and diagnostic studies of animals on farmsteads in certain regions.

\section{Results of research}

From 2006 to 2012, the specific animal health-care anti-brucellosis actions for the cases of brucellosis in cattle in the country were carried out in the form of systematic diagnostic studies and slaughter of positively reacting animals. However, veterinary reports data show that this method has not provided positive results and achievements in improving the brucellosis control has often been followed by periods of brucellosis unsafe situation [5].

Given this situation, in 2012, some livestock farms of the republic started using vaccine obtained from strain B. abortus 82, 19, 75/79-AV, RB 51 as a part of the set of cattle brucellosis control measures.

In the beginning, we have analyzed the effectiveness of the use of live vaccines (manufactured in Russia) obtained from strains of B. abortus 19, 82 and 75/79-AV in combination with post-vaccination diagnostics of brucellosis in cattle in the farmsteads owned by Vostok-moloko Corporation in Eastern Kazakhstan in the period of 2012-2015.

Young animals of 3-4 months old were immunized with a vaccine obtained from the strain 82 (based on epizootological indications, it was a vaccine obtained from the strain 19). Heifers that were 2-3 months before their insemination and then cows with an interval of 1 year were reimmunized with a vaccine obtained from the strain 82 . In situations where the adult breeding livestock, including pregnant animals, had no immunity or their immunity was doubtful, the primary immunization of animals was carried out with non-abortive vaccine obtained from strain 75/79-AV, and subsequently reimmunized with a vaccine obtained from strain 82 with an interval of 1 year.

Until September 2012, three other farmsteads owned by the corporation (Ukrainka, Donskoye, and Shemonaikha), demonstrated a number of their animals that were seropositive for brucellosis. Veterinary healthcare measures in such herds went practically without the use of anti- 
brucellosis vaccines - in Kazakhstan, vaccination against brucellosis with live vaccine obtained from strain 82 was officially banned, and only in 2011, this ban was partially lifted to allow the use of such vaccination for young animals. In September and November 2012, there were three comprehensive diagnostic studies of brucellosis in cattle blood serum carried out at each of the abovementioned farmsteads. The first study with applied set of test methods (AT, complement fixation tests [CFT]) showed $2.2 \%$ of animals that were epizootically dangerous for brucellosis; where $0.8 \%$ were revealed through IDT with 0-PS antigen (indicator of the greatest epizootic hazard); the second study showed $1.8 \%$ and $0.8 \%$ and the third $-0.1 .3 \%$ and $0.3 \%$, respectively. Such results have led to a decision to use vaccines within the reasonable limits, starting December 2012. The first study of brucellosis in immunized breeding livestock after vaccination was carried out in February - March 2013 and that is 2-3 months following the immunization. Such tests allowed provoking hidden forms of brucellosis to show up in $3.2 \%$ of epizootically dangerous animals from the numbers of tested cattle, including $1.3 \%$ revealed with the use of IDT. There were three more comprehensive studies of brucellosis in adult breeding livestock conducted in 2013. These studies have revealed $1.7 \%$ epizootically dangerous animals from the mean number of tested animals, with $0.7 \%$ of them diagnosed through IDT with 0-PS antigen.

There were two other studies conducted in 2014: 0.7\% epizootically dangerous animals were detected out of the mean number of animals tested, with $0.1 \%$ of them diagnosed through IDT with O-PS antigen. All epizootically dangerous animals were duly isolated from the common herd and sent for slaughter.

The presence of positive and tentative reactions during the AT and CFT with the S-antigen in low titers indicates the post-vaccination nature of these reactions. This is confirmed by the practical absence of positives in IDT among the tested animals, which acts as an indicator of the high epizootic hazard, as well as by the presence of positives in CFT with $\mathrm{R}$-antigen in high titers that acts as an indicator of the vaccinial nature of the reactions. Thus, the system of anti-brucellosis measures was introduced in brucellosis threatened and unsafe farmsteads of "Vostokmoloko" Corporation by ensuring permanent immunity in herds of cattle using live vaccines obtained from strains 19, 82 and 75/79-AV and post-vaccination diagnostics with rational use of their provocative properties.

This helped to decrease the number of revealed epizootically dangerous animals to few individual cases in certain cattle herds within 2-year period.

The live anti-brucellosis vaccine $B$. abortus obtained from the strain RB 51 (manufactured in the USA) is registered in the Register of veterinary products of Kazakhstan since 2012.

It is prepared from R-forms of Brucella, therefore it does not cause the formation of S- antibodies in the immunized organism, so the study of vaccinated animals may be carried out at any time after immunization and that favorably distinguishes it from other vaccines [6-11].

The vaccine for prevention of brucellosis in cattle is being used in some farms of Kazakhstan since 2012.

We have analyzed the effectiveness of using this and other live vaccines obtained from strains of B. abortus 82 and 75/79-AV, in some regions of Kazakhstan in 2012-2014.

Before the vaccine was applied, the animals were subjected to a single serological test (except for animals in North Kazakhstan that were tested twice), and the positives were isolated from the herd; the rest of the animals were immunized with above-mentioned vaccines in accordance with their manuals. Subsequent studies of vaccinated animals were carried out in 7-8 months following the immunization. To analyze the effectiveness of vaccination in the abovementioned areas, we used the data provided by the regional veterinary services and diagnostic data from our own research.

Table 1 shows the information about vaccination and diagnostic testing of cattle in East Kazakhstan, West Kazakhstan, North Kazakhstan, Kostanay, and Karaganda region for the period of 2012-2014.

As it can be seen in Table 1 in the period of 2012-2014, there were 15428 animals immunized with different anti-brucellosis vaccine in Eastern Kazakhstan, where 8491 animals were immunized with the vaccine obtained from the strain B. abortus 82, 1715 animals - with the vaccine obtained from the strain 75/79-AV and 2552 animals with the vaccine obtained from the strain RB 51. The diagnostic serology tests conducted in 2013, which is 7-8 months after immunization, has revealed the positive results for brucellosis in the 19 animals vaccinated with strain 82 based vaccine (infection rate of 1.06\%) and in 6 animals vaccinated with the strain RB 51 based vaccine (infection rate of $0.7 \%$ ).

The vaccine that proved to be most effective was the one that was obtained from the strain $75 / 79-\mathrm{AV}$, since the 3 years long studies of 1715 animals, vaccinated with this vaccine, and has not revealed any positive cases.

In 2014, the vaccine obtained from the strain RB 51 was used in Northern Kazakhstan, Western Kazakhstan, Karaganda, and Kostanay regions for the prevention of brucellosis in cattle.

The post-vaccination diagnostic studies conducted 7-8 months after the animals immunization with the vaccine obtained from the strain RB 51 have detected the infection with brucellosis in Karaganda region - 3.5\%, Western Kazakhstan - 7.1\%, Kostanay region - 23.0\%, which points at the lowest immunological efficacy of this vaccine.

To determine the effectiveness of vaccines, we have analyzed each of the above-mentioned cases of use of strain RB51 based vaccine for prevention of brucellosis in cattle.

Thus, in 2014 in the North-Kazakhstan region, rayon named after M. Zhumabaev, in Chistovskoe LLP farmstead a live lyophilized vaccine obtained from the strain B. abortus RB-51 (manufactured in the USA) was used against cattle brucellosis. Before the vaccine application, the rate of infection with brucellosis in this sector was $4.4 \%$.

For the period from $04 / 30 / 2014$ to $06 / 17 / 2014$ a total of 235 animals were vaccinated, the heifers that were born the same year and prepared for tupping. Before vaccination, all the animals have passed through diagnostic tests for brucellosis twice. The vaccine was administered to negatively reacting animals only and after separating the positively reacting animals from the common herd. The tests on these animals that were carried out 7 months after immunization all showed negative for brucellosis, indicating the absence of brucellosis infection in the animals that were subject to potential infection.

Before the vaccine use in the Karaganda region, the level of infection with brucellosis in the cattle at Eskene farmstead, Zhanaarka rayon and in Kaynar farmstead, Nura rayon was $1.3 \%$ and $4.9 \%$, respectively. After a single serological test carried out, 1200 animals at these farms were immunized in 2014 with the vaccine obtained from the strain RB 51. Further studies of these animals 1 year after vaccination showed that brucellosis infection was at $3.5 \%$, which also proves the low efficacy of the vaccine. There were no registered cases of animal abortions during this period.

A complicated epizootic situation with brucellosis in cattle has emerged in 2014 in one of the Western Kazakhstan farmsteads where the herd infection level has made more than $10 \%$. In August 2014, after the serology testing of the entire herd and separation of positively reacting animals the animals were vaccinated with the vaccine RB 51 in the following proportion: 160 heifers born in 2013 and 205 calves born in 2014 aged 3-5 months. Subsequent serological study was conducted 
Table 1: Data on vaccination and diagnostic studies of cattle in the Republic of Kazakhstan in 2012-2014

\begin{tabular}{|c|c|c|c|c|c|}
\hline \multirow[t]{2}{*}{ Regions, year } & \multirow[t]{2}{*}{ Type of vaccine } & \multirow[t]{2}{*}{ Quantity of vaccinated animals } & \multicolumn{3}{|c|}{ Results of serology assays after vaccination } \\
\hline & & & Tested & Positives & $\%$ of infection acquired \\
\hline Eastern Kazakhstan, 2012-2014 & Strain 82 & 8491 & 6084 & & \\
\hline 2012 & $-/-/-/-$ & 534 & 149 & - & - \\
\hline 2013 & $-/-/-/-$ & 2771 & 1790 & 19 & 1.06 \\
\hline 2014 & $-/-/-/-$ & 5186 & 4145 & - & - \\
\hline Eastern Kazakhstan, 2012-2014 & Strain 75/79-AB & 1715 & 840 & - & - \\
\hline 2012 & $-/-/-/-$ & 97 & 97 & - & - \\
\hline 2013 & $-1-/-/-$ & 1185 & 743 & - & - \\
\hline 2014 & $-/-/-/-$ & 433 & - & - & - \\
\hline Eastern Kazakhstan, 2012-2014 & Strain RB 51 & 2552 & 1561 & - & - \\
\hline 2012 & $-/-/-/-$ & 93 & 54 & - & - \\
\hline 2013 & $-/-/-/-$ & 1580 & 847 & 6 & 0.7 \\
\hline 2014 & $-1-/-/-$ & 879 & 660 & 13 & 1.9 \\
\hline North Kazakhstan, 2014 & Strain RB 51 & 235 & 235 & - & - \\
\hline Karaganda region, 2014 & Strain RB 51 & 1200 & 1200 & 42 & 3.5 \\
\hline Western Kazakhstan, 2014 & Strain RB 51 & 365 & 365 & 26 & 7.1 \\
\hline Kostanay region, 2014 & Strain RB 51 & 870 & 870 & 202 & 23.0 \\
\hline Total & & 15428 & 11155 & 308 & 2.8 \\
\hline
\end{tabular}

in March 2015 and revealed the following ratio of positively reacting animals: 18 heifers born in 2013, 2 bull calves born in 2014 and 6 heifers born in 2014. It should be mentioned that 2 of the heifers born in 2013 had abortions. A total of 26 animals showed a positive reaction.

Analysis of the cases of use of the vaccine obtained from the strain RB 51 may lead to a conclusion, that 7 months after immunization, 7.1\% animals showed positive results for brucellosis, with 2 heifers having the abortion. These data demonstrate low immunological efficacy of that vaccine.

A study conducted in August 2014 in Tobolsk-1 LLP, Dosovka village, Denisovsk rayon of Kostanay region, for brucellosis in 603 animals (Rose Bengal test [RBT], CFT, AT), have showed 13 positives while the percentage of infection has made $2.1 \%$. It is important to mention that the results of tests indicate that no any other actions were made with regard to 13 positively reacting animals except for sending them to slaughter. Then, 7 months later, in March 2015, the 545 animals on this farm were (without approval and notification to the local veterinary inspection body) immunized with the anti-brucellosis vaccine obtained from B. abortus RB-51 (manufactured in the USA). Moreover, these animals were not tested for brucellosis before vaccination. In addition, RB 51 vaccine was applied to325 more animals on this farmstead which makes total of 870 vaccinated animals.

Later, in June 2015 (3 months after vaccination), during scheduled diagnostic serological tests for brucellosis in 926 animals in the Dosovka farmstead, 202 cattle had their blood serum showing positive results during the conventional serological tests (RBT, CFT, AT), including: 112 cows and 90 young animals born in 2015, while the percentage of infection has made $21.8 \%$. However, the management of Tobolsk LLP took an unreasonable decision not to send the brucellosis positive animals to the slaughter, wrongly thinking that these reactions are of post-vaccination nature and instead have sent the blood serum of 112 cows for differential assays in RSE "National Reference Veterinary Center," the KZ Ministry of Agriculture (hereinafter RSE nonresident violator compact [NRVC]).

As for the interpretation of test results obtained by RSE NRVC who have carried out enzyme-linked immunosorbent assay (ELISA) with the test-kit for the detection and differentiation of antibodies to the $\mathrm{S}$ - and R-forms of the agents of brucellosis, it should be noted that 93 blood serum samples showed the presence of specific antibodies to brucellosis S-shape antigens, which makes these animals referred to as the infected with brucellosis and only 5 samples had R-shape antibodies. Analysis of the test results for 112 seropositive blood serum samples in IDT with O-PS antigen showed that more than $48 \%$ of the tested animals had positive reaction for brucellosis (Test Report \#138-15-Ф, dated 07/10/2015).

\section{RESULTS AND DISCUSSION}

Thus, the tests result obtained both from ELISA and from IDT with O-PS antigen suggest a contagious and not post-vaccination nature of demonstrated positive serological reactions.

We believe that in this case, the massive detection of positively reacting animals during officially conducted tests for brucellosis, are related to the provoking effect of the vaccine obtained from the strain RB-51, which is prepared from non-agglutinogenic R-strain of Brucella. Given the fact that the animals were not tested for brucellosis before the vaccination, the introduced R-vaccine has provoked a "silent" course of brucellosis, which has caused massive positive reactions to brucellosis.

Later on, in 2015, for the purpose of a study of the optimal scheme of using the vaccine, we conducted on-site tests in Eskene farmstead, Zhanaarka rayon of Karaganda region and in Asem farmstead, Akzhaik rayon of Western Kazakhstan, by immunizing cattle with the vaccine obtained from the strain RB 51. We selected certain quantity of animals from each of these two farms from the numbers of breeding livestock having the similar epizootic status of the infection with brucellosis. 105 cows in the age of 3-5 years old were tested for brucellosis in July 2015 in the Eskene farmstead, of Zhanaarka rayon of Karaganda region; 4 of these cows (3.8\%) had positive reactions for brucellosis. 96 cows in the age of 3-5 years old, tested in Asem farmstead, Akzhaik rayon of Western Kazakhstan, had 3 of them (3.1\%) reacting positively before vaccination.

After separation of positive animals from the herd, the rest of the animals were immunized with the vaccine obtained from the strain RB 51 in accordance with its user manuals. A month after immunization the animals at both farmsteads were re-examined with the conventional tests (AT, CFT, RBT) for brucellosis, and this time each of the herds showed 2 animals positively reacting to the brucellosis - these animals were also separated from the herd. During the winter period of 2015-2016, when the cattle is kept in a stall, these animals had no any cases of abortion or other manifestations of brucellosis infection.

Routine serological testing of vaccinated animals was carried out 9 months after immunization (May 2016) with no any observed cases of positively reacting animals.

The results of these tests prove that studies of animals 1 month after the vaccination have helped to further identify the animals with hidden form of brucellosis provoked by R-vaccine, which explains the favorable outcome of the research. 


\section{CONCLUSIONS}

A positive experience in implementing anti-brucellosis control in cattle farmsteads owned by the Vostok-moloko Corporation in Eastern Kazakhstan, based on the rational use of live vaccines obtained from the strain B. abortus 19, 82 and 75/79-AV, as well as post-vaccination diagnostics tools in combination with the common veterinary, sanitary, organizational and economic measures, justifies the need of applying this scheme in other farms of the Republic of Kazakhstan. The above-described scheme of anti-brucellosis activities ensures effective control of epizootic process of brucellosis by establishing "permanent" (continuous) immunity and permanent control of epizooty in brucellosis threatened/unsafe herds $[12,13]$. As a result, a long-term "biological balance" was created and maintained at all levels of "animals - organisms" systems, helping to prevent the formation of epizootically dangerous options of the pathogen.

A single-time serological testing for brucellosis in cattle before immunization with B. abortus vaccine based on the strain RB 51 and separation of positively reacting animals does not provide accurate detection of all infected animals in the herd. Before use the vaccine, the testing of animal for brucellosis should be carried out at least 2 times, with a mandatory separation of positive animals from the herd. The use of B. abortus vaccine obtained from the strain RB 51 in brucellosis unsafe herds did not ensure the formation of the strong immunity. The level of infection with brucellosis in herds of animals immunized 7-8 months ago was varying from $0.7 \%$ to $7.1 \%$. To enhance the effectiveness of B. abortus vaccine, obtained from the strain RB 51, it is recommended to use it for provoking the latent brucellosis properties.

\section{REFERENCES}

1. Ivanov NP. Brucellosis in Animals and Measures of its Control. Almaty: Zoology; 2007. p. 617-25.

2. Abdrakhmanov S, Abutalip A, Baramova SA. Evaluation of Epizootic Process and Prediction of the Geographic Spread of Brucellosis in
Farm Animals. Uralsk: Materials Medical Research and Production Center, West Kazakhstan Agrarian Technical University Named after Zhangirhan; 2012. p. 141-6.

3. Sultanov A, Ten V, Mikhalev N. Some of the changes in strategies for the elimination of brucellosis in animals. Actual Problems of Animal Diseases in Modern Conditions: The Materials of International Scientific and Practical Conference, Dushanbe; 2003. p. 72-5.

4. Mustafin M. Specific prevention of brucellosis in cattle. J Vet Sci 2004;13:50-62.

5. Sultanov A, Baramova S, Abutalip A, Ospanov Y. Epizootic Situation with Animal Brucellosis in the Republic of Kazakhstan. Vol. 11. Almaty: Collection of Scientific Materials by KazNIVI; 2015.

6. Schurig GG, Sriranganathan N, Corbel MJ. Brucellosis vaccines: Past, present and future. Vet Microbiol 2002;90(1-4):479-96.

7. Lord VR, Schurig GG, Cherwonogrodzky JW, Marcano MJ, Melendez GE. Field study of vaccination of cattle with Brucella abortus strains RB51 and 19 under high and low disease prevalence. Am J Vet Res 1998;59(8):1016-20.

8. Poester FP, Gonçalves VS, Paixão TA, Santos RL, Olsen SC, Schurig GG, et al. Efficacy of strain RB51 vaccine in heifers against experimental brucellosis. Vaccine 2006;24(25):5327-34

9. Ashford DA, di Pietra J, Lingappa J, Woods C, Noll H, Neville B, et al. Adverse events in humans associated with accidental exposure to the livestock brucellosis vaccine RB51. Vaccine 2004;22(25-26):3435-9.

10. el Idrissi AH, Benkirane A, el Maadoudi M, Bouslikhane M, Berrada J, Zerouali A. Comparison of the efficacy of Brucella abortus strain RB51 and Brucella melitensis Rev 1 live vaccines against experimental infection with Brucella melitensis in pregnant ewes. Rev Sci Tech 2001;20(3):741-7.

11. Diptee MD, Adesiyun AA, Asgarali Z, Campbell M, Adone R. Serologic responses, biosafety and clearance of four dosages of Brucella abortus strain RB51 in 6-10 months old water buffalo (Bubalus bubalis). Vet Immunol Immunopathol 2006;109(1-2):43-55.

12. Mondal I, Sanyal S, Das S. Coagulation profile in patients suffering from acute brucellosis. Asian J Pharm Clin Res 2013;4:179-80.

13. Gupta A, Patel SS, Langute SM, Kolekar MR, Bhosale HP, Shinde SK, et al. Bacterial diseases of livestock animals and their impact on human health. Asian J Pharm Clin Res 2016;6:8-11. 